\title{
Laboratory costing system based on number and type of test: its association with the Welcan workload measurement system
}

\author{
I F Tarbit
}

\begin{abstract}
A laboratory costing system which recovers all costs against tests, rather than using both test and request charges, was developed. Methods of recovering costs of routine and emergency services, of capital investment in equipment, of instrument maintenance costs and of general hospital overheads were considered. The Welcan unit system of workload measurement was applied to a range of test procedures. Both the Welcan unit value and unit value adjusted for calibration and quality control (Welcan based weighting) correlated only moderately with locally derived analytical time per test and correlated poorly with direct analytical cost per test. The correlation of direct analytical cost per test with total cost per test was much stronger than that of analytical time per test with direct analytical cost per test.

The data suggest that neither Welcan unit values, Welcan based weightings, nor locally derived analytical time per test can truly reflect total resource consumption for the provision of a range of test procedures. This factor should be borne in mind when applying operational or performance indicators based on Welcan units.
\end{abstract}

In 1986 I described a clinical biochemistry costing system which differentiated between test costs and charges levied against requests. ${ }^{1}$ This paper describes a laboratory costing system based on tests-number and type-and the rationale of loading all costs on to tests in relation to the provision of pathology services which have to conform to contract.

Current National Health Service performance indicators based on unweighted requests as a measure of laboratory workload have been criticised as being too crude a measure of laboratory activity. The Welcan workload measurement system, ${ }^{2}$ developed from the Canadian schedule of unit values for clinical laboratory procedures, ${ }^{3}$ attempts to provide a series of relative weighted procedure values reflecting labour input. It is proposed that this measurement system should be in national use by 1990 as a basis for revised performance and operational indicators.

This study compares information on the allocation and use of resources by adapting the Welcan workload measurement system to that generated from a comprehensive laboratory costing system.

\section{Methods \\ THE COSTING SYSTEM \\ Direct materials costs}

As described previously ${ }^{1}$ reagent costs per sample were determined for each analysis type by assessing expenditure on each reagent over an extended time period and dividing this figure by the number of patient samples analysed in the same period. Thus reagent costs of calibration, quality control, and repeat analyses are built into a true cost per patient sample.

Other consumables such as pipette tips, sample cups, calibration and control sera were assigned a unit cost from invoice records. Multiplying unit cost by the unit volume used per test gave the specific consumable cost for that test.

Inhouse maintenance costs of major instrumentation were assessed over an extended time period and allocated uniformly over all samples analysed on that instrument in the same period.

\section{Direct labour costs}

These were maximised where possible. Several elements were included.

Analytical costs: Section heads accurately timed the average batch of analyses, including pre-analytical preparation of materials and recording of post-analytical results. Time per average batch divided by the number of patient samples (rather than tests) within the batch gives a standard time (in minutes) per sample.

A composite labour rate for trainee MLSO's and MLSO's grades 1 and 2 was used in most instances. This composite labour rate (pounds sterling per minute) is weighted to reflect numbers of each grade in post. At the time of writing the current rate was $£ 0.0963$ per minute.

Sample preparation costs: Labour costs for preanalysis steps such as specimen reception and numbering, centrifugal separation of plasma from red cells, and aliquoting into various sample tubes were allocated as direct costs. Employing three trainee/grade 1 MLSO's to handle 154073 requests, generating 882330 tests in 1988, resulted in a sample preparation labour rate of $£ 0.0298$ per test. 
Computer input costs: Computer input of data is an essential element of the analytical process and the labour costs are attributed as direct costs in this system. This work is carried out by two part-time whole time equivalent of staff (WTE) clerk/typists and one medical laboratory assistant (MLA). Total salaries involved are recovered as a uniform rate levied for each test. This was $£ 0.0172$ per test at the time of writing.

Supervisory costs: Senior staff time taken up with direct supervision of the analytical function is also seen as a charge justifiably levied against direct costs. This supervisory time is more difficult to quantify and considered estimates were used. Eighty per cent of chief MLSO costs and $30 \%$ of senior chief MLSO costs were recovered in this way.

Medical/scientist time taken up in vetting and validating reports before issue to the wards was also recovered as a direct cost. An average of $30 \%$ of medical and scientist costs were levied as a direct charge against tests. At the time of writing this gave a supervisory cost of $£ 0.0973$ per test (including medical costs).

\section{Indirect materials costs}

The product of test workload multiplied by direct materials cost gives materials recovery for each procedure. Summing for each test gives total materials recovery as direct costs. This falls short of total non-staff budget recovery. This shortfall includes costs of unaccounted reagent wastage, materials used in training, method development and laboratory or ward based research projects.

Total non-staff budget recovery is achieved by dividing this residue of materials costs by total test workload to give an indirect materials cost per test. This stood at $£ 0.0864$ at the time of writing.

Table 1 Example cost analysis for triglyceride assay (Cobas Mira) and CK-MB assay (Cobas Bio)

\begin{tabular}{|c|c|c|}
\hline & Triglycerides & $C K-M B$ \\
\hline $\begin{array}{l}\text { Direct materials costs } \\
\text { Consumables } \\
\text { Reagents } \\
\text { Calibrant and QC material }\end{array}$ & $\begin{array}{l}0.0589 \\
0.1150 \\
0.0710\end{array}$ & $\begin{array}{l}0.2166 \\
0.5570 \\
0.1960\end{array}$ \\
\hline $\begin{array}{l}\text { Indirect materials costs } \\
\text { Standard budget recovery charge }\end{array}$ & $0 \cdot 2449$ & 0.9696 \\
\hline Materials total cost & $0 \cdot 3286$ & 1.0533 \\
\hline $\begin{array}{l}\text { Direct labour costs } \\
\text { Analytical cost: (minutes) (rate: } £ 0.0963 / \text { minute) } \\
\text { Sample preparation } \\
\text { Computer request input } \\
\text { Supervisory costs (including medical element) }\end{array}$ & $\begin{array}{l}0.1522(1.58) \\
0.0298 \\
0.0172 \\
0.0973\end{array}$ & $\begin{array}{l}0 \cdot 6740(7 \cdot 0) \\
0 \cdot 0298 \\
0 \cdot 0172 \\
0 \cdot 0973\end{array}$ \\
\hline \multirow{3}{*}{$\begin{array}{l}\text { Indirect labour costs } \\
\text { Advisory, management, service development } \\
\text { Standard MLSO budget recovery charge }\end{array}$} & $0 \cdot 2965$ & $0 \cdot 8183$ \\
\hline & $\begin{array}{l}0.1584 \\
0.0753\end{array}$ & $\begin{array}{l}0.1584 \\
0.0753\end{array}$ \\
\hline & $0 \cdot 2337$ & $0 \cdot 2337$ \\
\hline $\begin{array}{l}\text { Labour total cost }(\mathscr{L}) \\
\text { Total test charge }(\mathscr{\complement})\end{array}$ & $\begin{array}{l}0.5302 \\
0.8588\end{array}$ & $\begin{array}{l}1.0520 \\
2 \cdot 1053\end{array}$ \\
\hline
\end{tabular}

Indirect labour costs

Multiplying a particular test workload by its direct labour cost and summing for each test gives an assessment of labour recovery as direct charges. The shortfall from the total staff budget (including medical staff costs) represents indirect labour costs and relates to staff time taken up with such vital functions as advising clinicians, training, teaching, service development and laboratory management and secretarial functions.

This is recovered in two ways. Secretarial salaries, percentages of senior chief and chief MLSO salaries not assigned to direct test supervision, and those percentages of medical and scientist salaries not assigned to result vetting are recovered as a standard charge against each test- $£ 0 \cdot 1584$. The residue of MLSO salaries up to senior MLSO grade not recovered as direct labour is apportioned as a standard charge across each test- $-£ 0.0753$.

Example cost breakdowns for triglyceride assay on the Roche Cobas Mira and CK-MB isoenzyme assay on the Roche Cobas Bio are provided in table 1 .

\section{Other cost elements}

Costs of "on-call" service are also recovered against tests such that service users are able to make a direct comparison of the relative costs of "on-call" and routine services. The total cost of "on-call" service including call payments, standby payments, and travel expenses was $£ 61253$ in 1988-89.

A standard labour charge is levied per emergency test derived by dividing the total staffing cost of $£ 61253$ by the emergency workload of 73747 tests, giving an emergency service labour charge of $£ 0.8306$ per test. This is reassessed annually.

Direct materials costs but not indirect materials costs (fully recovered against routine tests) are also charged against each emergency test. Thus the charge for an "on-call" sodium estimation comprises $£ 0.8306$ labour cost and $£ 0.0563$ direct materials cost, giving a total cost of $£ 0.8869$ compared with a routine service cost of $£ 0.5568$. An urgent electrolyte profile of six tests processed on the Beckman Sychron CX3 during normal working hours therefore carries a total cost of $£ 3.663$, while the same profile done on an "on-call" basis incurs a total cost of $£ 5 \cdot 524$. Following the publication of the White Paper Working for Patients hospitals will be expected to recover both capital and revenue costs against service delivery and will have to incorporate these elements into a full laboratory costing system.

Hotel and service costs $(£ 55986$ for Freeman Hospital clinical biochemistry department in 1988-1989) can be levied as a standard charge against each test $(£ 0.063$ per test for an 882330 test workload at Freeman Hospital).

Annual equipment maintenance costs can also be recovered as a standard test charge against those tests done on a given instrument. For example, 1988-1989 maintenance costs for the Cobas Mira were $£ 2995$ and annual work- 
Figure 1 Scattergram showing correlation between Welcan based test weighting and test analytical time. $r=0.677, p<0.001$, $y=0.903 x+3.079)$

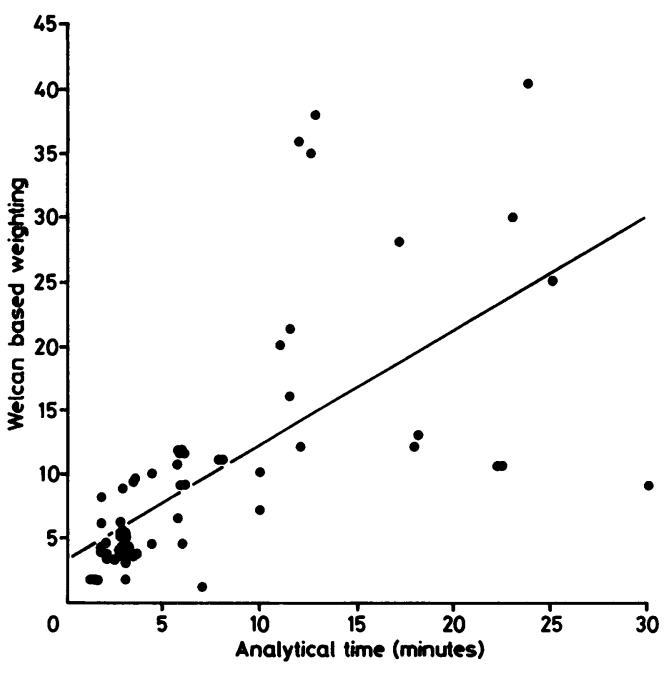

load comprised 14685 tests. Levying a standard charge across all test types, the equipment maintenance cost per test would be $\frac{£ 2995}{14685}=£ 0 \cdot 204$.

For recovery of capital investment in equipment two options might be considered.

(1) Apportionment of total equipment investment equally across all tests.

While many low cost items may stay in service for over 10 years, more expensive and sophisticated instrumentation cannot be expected to function reliably beyond seven to eight years, and the projected life expectancy of computer hardware is five years or less. Stillwell suggests that an estimated equipment life of $12-15$ years should be used, ${ }^{4}$ but this seems to be highly optimistic and a more conservative figure of seven years may be more realistic.

For the current equipment investment of £599018 in clinical biochemistry at Freeman Hospital, amortisation over seven years would require a recovery of $£ 85574$ in 1988-1989 against 882330 tests, giving a standard amortisation charge per test of $£ 0.0982$. This would be uplifted each year for prevailing inflation rates.

(2) Capital investment recovery could be loaded against those tests using that equipment.

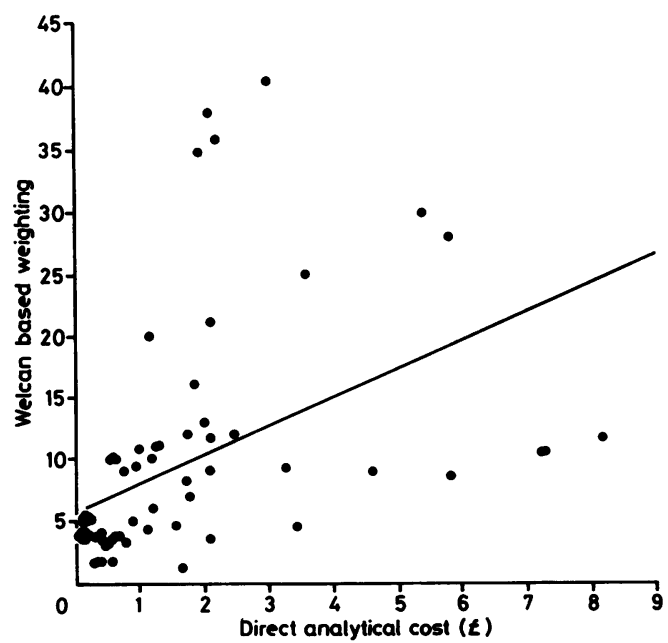

Recovery of investment in a main-line analyser like the Olympus AU5000 would require a return of $£ 179322$ over a seven year life, or $£ 25617$ per annum. In 1988-1989, the AU5000 workload was 658567 tests.

(a) A standard amortisation charge could be applied per test of

$$
\frac{£ 25617}{658567}=£ 0.0389
$$

This approach would leave a common core of equipment investment to recover against all tests, such as centrifuges and computer facilities. At Freeman Hospital, discounting equipment recoverable against specific tests leaves $£ 200991$ for recovery against all tests. Over a seven year amortisation period this creates an additional charge of $£ 0.0325$ per test giving a total amortisation charge on each AU5000 test of $£ 0.0714$.

(b) The amortisation charge could be weighted to those tests requested more frequently on this selective analyser. As $8.4 \%$ of total tests in 1988-1989 were for sodium assay whereas $5.9 \%$ were for calcium assay, amortisation charge to each test cost might be $£ 0.0424$ and $£ 0.0298$, respectively.

Adding the standard charge for recovery of investment in other equipment, the total amortisation charge on AU5000 tests would vary from $£ 0.0749$ for sodium to $£ 0.0623$ for calcium.

\section{MEASURES OF LABORATORY ACTIVITY}

The Welcan system, like the Canadian system, provides a schedule of unit values per procedure to reflect the average technical, clerical, and aide time required to perform a laboratory procedure. One Welcan unit is equal to one minute of technical, clerical, and aide time.

The Freeman costing system incorporates an element of analytical time per test related to calibrant and quality control sample testing: the Welcan system counts each of these samples as a separate item. Test analytical time in the Freeman system is therefore not directly comparable with the Welcan unit value for that test.

To make a valid comparison the following process was adopted. For each procedure the total Welcan units generated from the average batch including patient specimens, calibrants, and quality control samples was counted and divided by the number of patient samples in the average batch to obtain a relative Welcan based test weighting. Thus for glucose the average batch includes 37 patient specimens, six calibrants, and six quality control specimens. The Welcan unit value for glucose assay on the Cobas Bio is 3.0 , giving a total Welcan unit count of 147 units for this average batch and a Welcan based weighting of $\frac{147}{37}=4 \cdot 0$.

\section{Results}

Figs 1-4 compare Welcan based weighting with analytical time, Welcan based weighting with direct analytical cost, analytical time with direct analytical cost and direct analytical cost with total cost for 75 assays. Four outliers with 
Figure 3 Scattergram between test analytical time and direct analytical cost per test. $y=2.434 x+3.098$ ) showing correlation $(r=0.669, p<0.001$

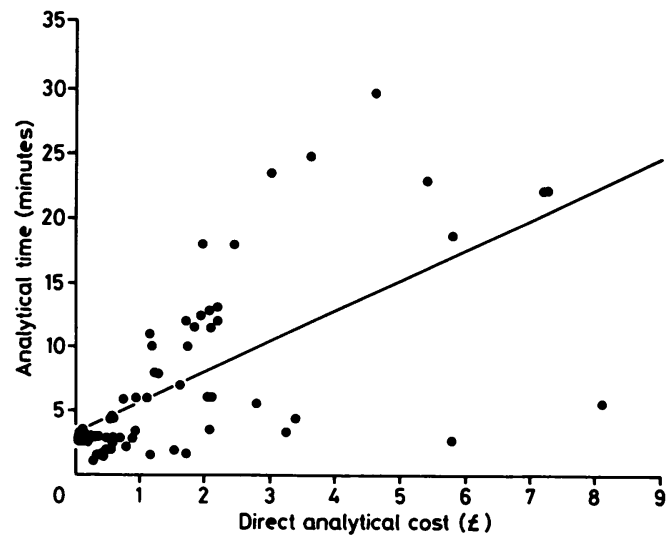

a Welcan based weighting greater than 45 or analytical time greater than 35.0 minutes, or both, were excluded to permit a less compressed representation of the bulk of data.

A full listing of Welcan Unit value, Welcan based weighting, analytical time, direct analytical cost and total cost per test for 79 assays can be obtained from the author.

\section{Discussion}

There are several reasons why a fully test based costing system is preferable to the mixed test/ request based costing system I described in 1986.

Following the publication of Working for Patients and its proposals on contracted services, laboratories will need to provide their clinical users with the costs of various procedures in a clear and simple manner. A glucose or cholesterol test cost must therefore be all encompassing-without hidden extras. Quoting separate request charges which do not relate directly to a particular test is merely confusing. Broughton and Hogan suggest that request "handling" charges are a more rational basis for allocating indirect costs. ${ }^{5}$ While this may be theoretically true, in practical terms one all encompassing cost per assay is more meaningful and understandable to service "purchasers".

The same argument holds for the future general practitioner budget holders. They will wish to have a clear and unequivocal list of

Figure 4 Scattergram showing correlation between total cost per test and direct analytical cost per test.

$(r=0.967, p<0.001$, $y=0.978 x+0.622)$

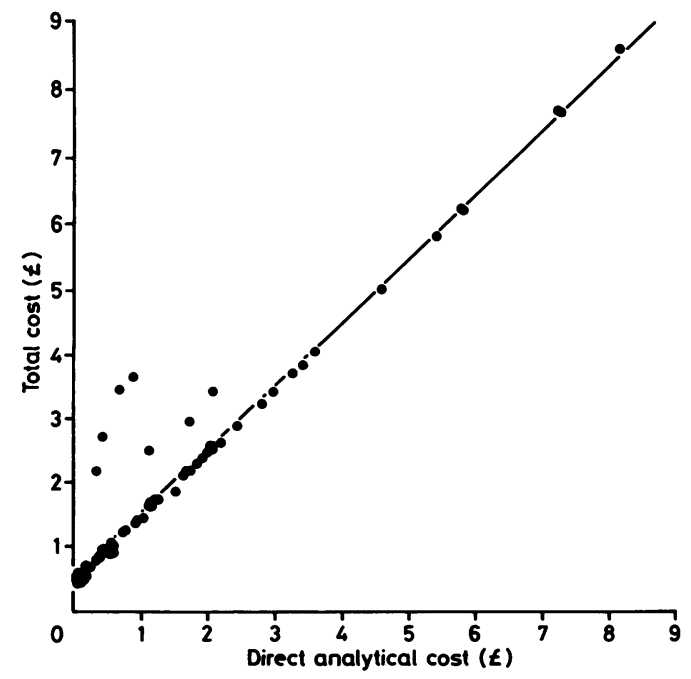

laboratory procedure charges presented to them. Similarly, if laboratory service providers explore possibilities for income generation outside their own district health authority or even the NHS, a clear statement of priced analytical services without the obfuscation of request charges will be required.

As Kreig et al point out, cost per test values when multiplied by their quantities, should match total cost to operate the laboratory. ${ }^{6}$ The system described here attempts to do that.

Broughton and Hogan suggest that allocating indirect costs to a request charge gives a clearer distinction between direct analytical costs related to workload, which are potentially controllable, and indirect costs which are less easily influenced by short term management. ${ }^{4}$ Certainly direct analytical costs should be identifiable. This can be done effectively within a global test cost as shown in table 1 , and test cost elements can be summarised in several ways to meet this requirement (table 2).

Broughton and Hogan's assumption that direct analytical costs are controllable by reducing workload needs qualification. Within direct costs there are usually fixed and variable elements. Costs of initial calibration may be fixed whether 10 or 60 tests are processed. With large selective analysers direct analytical labour per sample is often fixed whether two or 10 different analyses are done on each sample.

Methods of recovering "on-call" costs were examined earlier. Test based costing systems, however, should also reflect cost variances between batched and "stat" assays during the normal working day. Samples received requiring urgent electrolyte analyses are often handled on a different analyser from the bulk of electrolyte requests. Thus at Freeman Hospital an Olympus AU5121 analyser is used to process the bulk of electrolyte workload, while a Beckman Synchron CX3 provides urgent "stat" analyses. The costing methodology described in this paper reflects these differences (table 2). The economies of scale inherent in a high capacity analyser such as the Olympus AU5121 generate a lower direct labour cost per electrolyte of $£ 0 \cdot 188$ than for the Synchron CX3 where a higher direct labour cost per profile of $£ 0.353$ reflects extra labour costs incurred in providing faster turn round time per sample.

The standard cost profile for a particular test may not always reflect analytical situations. In certain circumstances a "stat" digoxin assay may be clinically justified. Such single samples require the same calibration and quality control as the usual batch of samples and therefore direct materials costs are higher. Use of analytical labour is less efficient and direct labour costs are consequently also higher. Direct analytical labour, materials, and total costs at Freeman Hospital for a batched digoxin assay are $£ 0.423, £ 2.972$, and $£ 3.395$, respectively while a "stat" digoxin assay incurs corresponding costs of $£ 2.022, £ 4.020$, and $£ 6 \cdot 042$.

With the advent of an increasing number of analysers providing a new range of "stat" analyses and a growing clinical requirement for 
Table 2 Example cost profiles highlighting direct and indirect cost elements

\begin{tabular}{|c|c|c|c|c|c|c|c|c|c|}
\hline \multirow[b]{3}{*}{ Test Procedure } & & & \multirow{3}{*}{$\begin{array}{l}\text { Total materials } \\
\text { cost }(£)\end{array}$} & \multicolumn{3}{|c|}{ Labour costs $(£)$} & \multirow{3}{*}{$\begin{array}{l}\text { Total direct } \\
\text { labour }(£)\end{array}$} & \multirow{3}{*}{$\begin{array}{l}\text { Total labour } \\
\text { cost }(£)\end{array}$} & \multirow{3}{*}{$\begin{array}{l}\text { Total } \\
\text { cost }(£)\end{array}$} \\
\hline & \multicolumn{2}{|c|}{ Materials costs $(£)$} & & \multicolumn{2}{|l|}{ Direct } & \multirow[b]{2}{*}{ Indirect } & & & \\
\hline & Direct & Indirect & & Analytical & Other & & & & \\
\hline Electrolyte profile (AU5000) & 0.495 & $0 \cdot 502$ & 0.997 & $0 \cdot 188$ & 0.865 & 1.402 & 1.053 & $2 \cdot 455$ & 3.452 \\
\hline Bone profile (AU5000) & 0.235 & 0.335 & 0.570 & $0 \cdot 101$ & 0.577 & 0.934 & 0.677 & 1.612 & $2 \cdot 182$ \\
\hline Liver profile (AU5000) & 0.267 & $0 \cdot 418$ & 0.685 & $0 \cdot 142$ & $0 \cdot 721$ & $1 \cdot 168$ & 0.863 & $2 \cdot 031$ & $2 \cdot 716$ \\
\hline Electrolyte profile (Synchron CX3) & 0.541 & 0.502 & 1.043 & 0.353 & 0.865 & 1.402 & $1 \cdot 218$ & $2 \cdot 620$ & 3.663 \\
\hline CK & 0.284 & 0.084 & 0.368 & $0 \cdot 193$ & $0 \cdot 144$ & 0.234 & 0.337 & 0.571 & 0.939 \\
\hline Glucose & 0.213 & 0.084 & 0.297 & $0 \cdot 164$ & $0 \cdot 144$ & 0.234 & $0 \cdot 308$ & 0.542 & $0 \cdot 839$ \\
\hline Cholesterol & $0 \cdot 174$ & 0.084 & $0 \cdot 258$ & 0.152 & $0 \cdot 144$ & 0.234 & $0 \cdot 296$ & 0.530 & $0 \cdot 788$ \\
\hline Blood gases & 0.493 & $0 \cdot 251$ & $0 \cdot 744$ & 0.617 & 0.433 & $0 \cdot 701$ & 1.050 & $1 \cdot 751$ & $2 \cdot 495$ \\
\hline TSH & $1 \cdot 498$ & 0.084 & 1.582 & $0 \cdot 216$ & $0 \cdot 144$ & $0 \cdot 234$ & 0.360 & 0.594 & $2 \cdot 176$ \\
\hline $\mathrm{T} 4$ & 0.712 & 0.084 & 0.796 & 0.304 & $0 \cdot 144$ & 0.234 & $0 \cdot 448$ & 0.682 & 1.478 \\
\hline Urine catecholamines & $0 \cdot 827$ & $0 \cdot 251$ & 1.078 & $1 \cdot 232$ & 0.433 & 0.701 & 1.665 & $2 \cdot 366$ & $3 \cdot 444$ \\
\hline
\end{tabular}

assays "on-demand", an increasing number of assays may have to be costed in both batch and "stat" modes.

This paper proposes a number of ways of recovering investment in laboratory equipment. Approach (2) might suggest that expensive equipment provision for a small number of assays may not be economically justifiable. New assays coming into service initially generate limited workloads. If such assays relied on sophisticated and expensive equipment, targeting equipment investment recovery at these assays might militate against their introduction into service at all. Table 3 shows comparative amortisation charges for approaches (1), (2a), and (2b) for a range of assays at Freeman Hospital. Given that approaches (2a) and (2b) are more complex in operation and may work against service development, approach (1) is probably the preferred method.

Fig 1 shows that Welcan based weighting correlates only moderately with the corresponding analytical time for that test procedure $(r=0.677, \mathrm{p}<0.001)$. The Welcan unit value per test also shows only moderate correlation with analytical time per test $(r=0.658, p<0.001)$. There are several reasons for this finding.

\section{SINGLETON VERSUS DUPLICATE ASSAYS}

The high precision thyroid stimulating hormone (TSH) assay in use enables singleton assay wheras $\mathrm{T} 3$ assays are run in duplicate. $\mathrm{T} 3$ analytical time per sample is therefore correspondingly higher. The Welcan system of unit values makes no allowance for replicate analyses and the increased analytical time incurred.

Table 3 Example test amortisation changes based on optional methods of capital investment recovery

\begin{tabular}{|c|c|c|c|}
\hline \multirow[b]{2}{*}{ Test (analyser) } & \multirow[b]{2}{*}{$\begin{array}{l}\text { Method (1) } \\
\text { Universal test } \\
\text { charge }(£)\end{array}$} & \multicolumn{2}{|c|}{$\begin{array}{l}\text { Method (2) } \\
\text { Charge targeted at tests using particular } \\
\text { analyser }\end{array}$} \\
\hline & & $\begin{array}{l}\text { (a) Universal } \\
\text { charge }(£)\end{array}$ & $\begin{array}{l}\text { (b) Weighted to } \\
\text { reflect workload } \\
\text { on instrument ( }(£)\end{array}$ \\
\hline $\begin{array}{l}\text { Plasma sodium (AU5000) } \\
\text { Plasma calcium (AU5000) } \\
\text { Plasma cholesterol (Cobas Mira) } \\
\text { Plasma urate (Cobas Mira) } \\
\text { Serum CK (Cobas Bio) } \\
\text { Plasma glucose (Cobas Bio) }\end{array}$ & $\begin{array}{l}0.098 \\
0.098 \\
0.098 \\
0.098 \\
0.098 \\
0.098\end{array}$ & $\begin{array}{l}0.071 \\
0.071 \\
0.323 \\
0.323 \\
0.318 \\
0.318\end{array}$ & $\begin{array}{l}0 \cdot 075 \\
0.062 \\
0 \cdot 439 \\
0 \cdot 227 \\
0 \cdot 410 \\
0.304\end{array}$ \\
\hline
\end{tabular}

\section{SAMPLE PREPARATION}

Welcan unit values assigned to particular instruments do not always reflect individual assay conditions. For the Cobas Bio, the first assay on a specimen carries a value of 3.0 whereas subsequent assays on the same specimen carry a unit value of 1.0 . Thus a batch of $\mathrm{CK}$ assays run on the Cobas Bio warrant a unit value of 3.0 per specimen. Subsequent assays for CK-MB on the same samples, however, are assigned a unit value of 1.0 . In practice immunoinhibition assay for CK-MB (Roche Isomune $\mathrm{CK}$ ) requires a preanalysis short incubation of two aliquots of sample and is attributed a longer analysis time of seven minutes compared with a $\mathrm{CK}$ analysis of two minutes.

\section{TECHNOLOGY}

A unit value of 7.0 is applied to all manual nonextraction direct radioimmunoassay (RIA) and non-RIA methods in the Welcan system and few automated immunoassay systems are listed. At Freeman Hospital a Stratus immunofluorescence analyser (Baxter Healthcare Ltd) is used for cortisol, ferritin, and digoxin assays. While this is not listed in the Welcan manual, unit values for assays on a similar instrument such as the Abbot TDX which is listed can be used. The Welcan system, however, does not incorporate unit values for other chemiluminescence or immunofluorescence systems, and all other assays such as those on Delfia (Pharmacia Ltd) or Amerlite (Amersham International plc) systems must be attributed the same unit value of $7 \cdot 0$.

In practice high precision $\mathrm{TSH}$ assay on the Amerlite system, performed once, has an analytical time of 1.8 minutes, whereas T3 assay done manually in duplicate has an analytical time of 3.5 minutes, and follicle stimulating hormone and luteinising hormone assays done in small labour intensive batches are assigned an analytical time of $\mathbf{2 2 . 4}$ minutes per specimen. In all cases the attributable Welcan unit value is $7 \cdot 0$, which fails to reflect the multiplicity of techniques available in the immunoassay field. Welcan unit values will probably continue to correlate poorly with local analytical practices in any area of rapidly changing technology.

Figs 2 and 3 show scattergrams of Welcan based weighting against direct analytical cost 
and test analytical time against direct analytical cost. Neither Welcan unit values, Welcan based weightings, nor test analytical time (and hence analysis labour cost) correlate well with direct analytical cost $(\mathrm{r}=0.378, \mathrm{p}<0.001$, $\mathrm{r}=0.445, \quad \mathrm{p}<0.001$ and $\mathrm{r}=0.669$, $\mathrm{p}<0.001$, respectively). Consumable costs often make a substantial contribution to many assay analytical cost profiles. Table 2 shows that direct materials costs per AU5000 profile vary considerably, the most expensive being the electrolyte profile in which costly enzymatic reagents are used for carbon dioxide and urea assays.

In immunoassays reagent costs can have a tremendous impact on direct analytical costs. T4, T3, and PTH have similar analytical times $(2 \cdot 7,3 \cdot 5$, and 2.9 minutes, respectively) and are assigned similar Welcan unit values and hence Welcan based weightings, but their direct analytical costs vary considerably $(£ 1 \cdot 02$, $£ 3 \cdot 25$, and $£ 5 \cdot 78$, respectively).

Finally, fig 4 shows that direct analytical costs show good correlation with total costs $(\mathrm{r}=0.967, \mathrm{p}<0.001)$-that is, the total test cost is not so distorted by indirect costs and overhead costs as to lose its relationship to actual analytical resource consumption.

While the Welcan system of unit values reflects labour use and will offer a considerable advance over current performance indicators using unweighted requests, it does not afford the same ability to assess accurately the resource consumption as is provided by a full costing system.

I am indebted to Mr A Marriner for his invaluable help in the detailed cost analysis presented here and to Ms J Bourne for her much appreciated secretarial assistance.

1 Tarbit IF. Costing clinical biochemistry services as part of an operational management budgeting system. J Clin an operational manage

2 WELCAN Workload measurement system for pathology: manual with schedule of unit values. 1988-89 Edition, Cardiff: DHSS, Welsh Office.

3 National Hospital Productivity Improvement Program. Canadian workload measurement system laboratory. A schedule of unit values for clinical laboratory procedures. 1986-87 Edition, Toronto, Ontario. Laboratory Workload Measurement Secretariat.

4 Stillwell JA. Costs of a clinical chemistry laboratory. J Clin Pathol 1982;34:589-94.

5 Broughton PMG, Hogan TC. A new approach to the costing of clinical laboratory tests. Ann Clin Biochem 1981; 18:330-42.

6 Kreig AF, Israel M, Fink R, Shearer LK. An approach to cost analysis of clinical laboratory services. Am J Clin Pathol 1978;69:525-36. 